\title{
DETERMINANTES GENÉTICOS Y SUS MECANISMOS DE ACCIÓN IMPLICADOS EN LA RESISTENCIA BACTERIANA A METALES PESADOS: UNA REVISIÓN.
}

\section{Genetic determinants and their mechanisms of action involved in bacterial resistance to heavy metals: a review.}

\author{
${ }^{1}$ Iza Guaman Joana Fernanda* (D), ${ }^{1}$ Recalde Moreno Celso Guillermo iD, ${ }^{2}$ Iza Guaman Cristian \\ Fabricio
}
${ }^{1}$ Escuela Superior Politécnica de Chimborazo, Facultad de Ciencias, Carrera de Ingeniería en Biotecnología Ambiental / Grupo de Energias Alternativas y Ambiente, Riobamba, Ecuador. ${ }^{2}$ Universitat Politécnica de Valéncia, Escuela Técnica Superior de Ingeniería del Diseño ETSID, Valencia, España.
*joana.iza@espoch.edu.ec

$\mathrm{R}$ esumen

Se identificó y describió los principales determinantes genéticos implicados en la resistencia bacteriana a metales pesados reportados en la literatura, así como sus usos a nivel biotecnológico y ambiental. Se llevó a cabo una revisión bibliográfica de información de los últimos 10 años encontrados en revistas con indexación SJR disponibles en las diferentes bases de datos; bosquejando la situación actual del conocimiento sobre el tema y realizando comparaciones cualitativas entre las investigaciones seleccionadas. Las bacterias se encuentran en constante evolución y se vuelven más resistentes gracias a la adquisición de genes que les permite hacer frente a los efectos tóxicos de los metales. Por ello, se compiló desde varios autores los determinantes genéticos de resistencia bacteriana para los metales, como el arsénico, mercurio, cromato y cadmio siendo respectivamente: ars, mer, chr, cad y czc; estos sistemas pueden localizarse en el cromosoma o plásmidos de las bacterias. Se describe el mecanismo de acción que codifica cada determinante, siendo la regulación y la desintoxicación enzimática los principales mecanismos. Finalmente, se comparó las aplicaciones biotecnológicas y ambientales de los determinantes, encontrándose un amplio uso en la construcción de biosensores y organismos genéticamente modificados.

Palabras Claves: Proteínas, enzimas, transcripción, transgénicos

\section{A bstract}

The main genetic determinants involved in bacterial resistance to heavy metals reported in the literature were identified and described, as well as their uses at biotechnological and environmental levels. A bibliographic review of information from the last ten years found in SJR indexed journals available in different databases was carried out, outlining the current state of knowledge on the subject and making qualitative comparisons between the selected researches. Bacteria are constantly evolving and becoming more resistant thanks, to the acquisition of genes that allow them to cope with the toxic effects of metals. Therefore, the genetic determinants of bacterial resistance to metals such as arsenic, mercury, chromate and cadmium were compiled from several authors, being respectively: ars, mer, chr, cad and $c z c$; these systems can be located in the chromosome and plasmids of bacteria. The mechanism of action encoded by each determinant is described, mainly regulation and enzymatic detoxification. Finally, the biotechnological and environmental applications of the determinants were compared, finding wide use in the construction of biosensors and genetically modified organisms.

Keywords: Proteins, enzymes, transcription, transgenics 


\section{INTRODUCCIÓN}

La contaminación ambiental generada por metales pesados es una de las principales preocupaciones a nivel mundial, altas concentraciones de estos elementos constituyen un grave problema, porque tienden a bioacumularse en el medio ocasionando daño celular y disfunción de los sistemas vitales en los organismos vivos que entran en contacto con ellos (1). Algunos metales como el Ni y Co, son esenciales a bajas concentraciones porque actúan como cofactores enzimáticos, pero los metales como el $\mathrm{Hg}, \mathrm{Cr}, \mathrm{Cd}, \mathrm{Pb}$ entre otros resultan tóxicos incluso a bajas concentraciones (2). Los entornos que presentan elevadas concentraciones de metales pesados constituyen un factor determinante en la evolución de la fisiología celular de ciertas especies bacterianas, ya que les permite desarrollar mecanismos para adaptarse y sobrevivir a estos medios cambiantes (3).

La asociación entre los contaminantes y la microbiota residente da lugar a una serie de procesos adaptativos que finalmente se expresan como mecanismos de resistencia, los cuales pueden ser codificados genéticamente, constitutivos o inducidos por la presencia del metal $(3,4)$. Los mecanismos de resistencia desarrollados por las bacterias se encuentran asociados a los denominados determinantes genéticos, los cuales pueden estar localizados en el cromosoma o en elementos genéticos móviles (2).

Las comunidades bacterianas en los sitios contaminados prosperan con la carga orgánica acumulada, productos tóxicos y metales pesados presentes en ellos. En dicho entorno, se espera que los microorganismos residentes posean genes que les permita degradar diversos contaminantes $(5,6)$. En la presente revisión se identificó y describió los principales determinantes genéticos implicados en la resistencia bacteriana a metales pesados, así como sus usos potenciales a nivel biotecnológico y ambiental.

\section{MATERIALES Y MÉTODOS}

\section{IDENTIFICACIÓN:}

Revisión bibliográfica de la literatura en las ba- ses de datos (Scimago Journal \& Country Rank; Redalyc; Scielo; Science Direct; Pubemed-NCBI, Nature, Oxford Academic, Plos, Elsevier, Scopus) a partir de un protocolo de busqueda exhaustivo. Se emplearon palabras clave como: ars operón, mer operón, chr operón, cad operón. Adicionalmente, se usaron operadores booleanos con las palabras clave como: ars operon AND mechanism, mer operon AND mechanism, cad mechanism OR expression, chr mechanism OR expression.

\section{TAMIZACIÓN:}

En esta etapa se aplicaron los criterios de inclusión: estudios dentro de revistas SJR de alto impacto, máximo 10 años de antigüedad, disponibles en el idioma inglés o español, con términos de búsqueda en el título o resumen y que contengan información referente a los determinantes de resistencia a metales pesados y sus mecanismos de acción.

\section{ELECCIÓN:}

Los criterios de exclusión correspondían a estudios que no contengan información relevante, se excluyeron investigaciones que no reporten información completa como: los genes que intervienen en el mecanismo de resistencia, aquellos que no especifiquen los productos de los genes implicados en la resistencia, información sin datos de los ensayos, aquellos que no muestren el efecto del metal sobre el crecimiento bacteriano.

\section{INCLUSIÓN:}

A los estudios seleccionados con las fases anteriores se les realizó la extracción de las siguientes variables: año y lugar de estudio, tipo de determinante genético, mecanismo de acción investigado y potencial biotecnológico y ambiental.

\section{ANÁLISIS DE LA INFORMACIÓN:}

En el análisis se realizó una síntesis cualitativa de la información.

\section{RESULTADOS}

DETERMINANTES DE RESISTENCIA AL ARSÉNICO Y SUS MECANISMOS

La organización de los operones ars varía entre 
las diferentes cepas bacterianas; sin embargo, existen genes centrales que siempre están presentes, un claro ejemplo es el operón arsRBC presente en Pseudomonas fluorescens MSP3, Staphylococcus aureus y Exiguobacterium antarcticum B7 $(1,2)$. Otro conjunto de genes presente en pocos genomas es el operón arsRDABC, este sistema se ha identificado en Acidiphilium multivorum AIU301 y en el plásmido R733 de Escherichia coli $(3,4)$. En todos estos genomas se evidencia la presencia del gen $\operatorname{ars} R$, no obstante, en Shewanella sp. O23s, arsR no se encuentra dentro del conjunto de genes ars, pero sigue regulando la expresión del operón (5). El producto génico de arsR es un represor transcripcional que responde a $\mathrm{As}^{3+}(6,7)$. En Rhodopseudomonas palustris CGA009 cuando el As ${ }^{3+}$ está ausente, ArsR se une a la región operador/promotor del operón $\mathrm{y}$, por lo tanto, reprime la transcripción de los genes aguas abajo. En contraste, cuando el $\mathrm{As}^{3+}$ está disponible, ArsR se une a él y pasa por cambios conformacionales, disociándose de la región operador/promotor $(8,9)$.

El gen más frecuente en el entorno es $\operatorname{ars} C$, su diversidad es producto de su funcionamiento en condiciones aeróbicas, anaeróbicas e incluso en amplios rangos de $\mathrm{pH}$ y temperatura, incluidos los extremos $(10,11)$. En contraste, se ha encontrado que $\operatorname{ars} B$ y acr3 están ampliamente distribuidos en el genoma de bacterias resistentes al arsénico (12). Por su parte, Li et al. (13) identifican al gen acr 3 como la principal bomba de salida de $\mathrm{As}^{3+}$ en la familia Burkholderiaceae. Asimismo, Bacillus sp. PVR-YHB1-1 y 58 de 98 aislados de Campylobacter sp., portan el gen acr3 $(14,16)$. Por el contrario, el gen ars $B$ ha sido identificado en Acidithiobacillus ferrooxidans AO1, Pseudomonas putida KT2440 y está presente en 76 de 98 aislados de Campylobacter sp. $(17,18,16)$.

Los productos génicos de los determinantes ars $B$ y acr3, llevan a cabo el mecanismo de expulsión del $\mathrm{As}^{3+}$ de la célula. El sistema de eflujo de $\mathrm{As}^{3+}$ en C. glutamicum se basa en dos permeasas Acr3 que no usan ATP para la liberación del $\mathrm{As}^{3+}$; sin embargo, trabajan juntas con diferente eficiencia (9). Villadangos et al. (19) mostraron que CgAcr3-1 es un antiportador que cataliza el intercambio de arsenito-protón con los residuos Cis129 y Glu305. Por el contrario, en Exiguobacterium sp., aislada de lagos andinos de altura de los Andes Sudamericanos, se ha identificado la bomba ArsB, la cual no contiene cisteínas conservadas y no hay tiolatos implicados en la translocación de $\mathrm{As}^{3+}(2,19)$

Los genes ars $A$ y ars $D$ están presentes en el genoma de O. tritici SCII24T, A. multivorum AIU301 y en E. coli. En la cepa AIU301, se ha demostrado la función reguladora del gen $\operatorname{ars} D(15,18,4$, 20). Por otra parte, en Bacillus sp. PVR-YHB1-1 y CDB3, este par de genes siempre están juntos, porque presentan funciones interrelacionadas, es decir, el producto génico de ars $D$ funciona como una metalochaperona, que transfiere arsénico al gen $\operatorname{ars} A$ y activa la bomba de salida de $\mathrm{As}^{3+}(20$, $14,21,22)$.

Los operones ars confieren resistencia al arsénico inorgánico, pero también pueden acoplarse con otros genes ars para permitir la desintoxicación de los organoarsénicos $(23,4)$. Por otro lado, el gen ars $M$ de R. palutris CGA009 permite la generación de compuestos metilados, en esta cepa, arsM se induce por $\mathrm{As}^{3+}$ (8). Firrincieli et al. (24) han identificado el gen arsI en varias cepas de Rhodococcus sp., principalmente, en Rhodococcus aetherivorans BCP1 (21). La actividad de ArsI depende de $\mathrm{Fe}^{2+}$ y usa $\mathrm{O}_{2}$ para la ruptura del enlace, la unión de $\mathrm{O}_{2}$ a $\mathrm{Fe}^{2+}$ permite la transferencia de electrones, convirtiendo el $\mathrm{O}_{2}$ en un anión superóxido $\left(\mathrm{O}_{2}\right)^{-2}$ que ataca a los organoarsénicos y forma un intermedio alquilperoxo- $\mathrm{Fe}^{2+}$, rompiendo el enlace $\mathrm{C}-\mathrm{As}$ (25).

El gen $\operatorname{arsH}$ está presente solamente en bacterias aeróbicas, como O. tritici SCII24T y A. ferrooxidans $\mathrm{AO} 1(26,15,27)$. Por otro lado, Shen et al. (16) identifican el gen ars $P$ en 54 aislados de Campylobacter jejuni, y evidencia la presencia de $\operatorname{ars} P$ en bacterias aeróbicas, no obstante, también está presente en bacterias anaeróbicas (26). Chen et al. (29) proponen un mecanismo llevado a cabo por ArsH: la reacción reductora consiste en la formación de un complejo FMN-NADPH oxidado seguido de una transferencia de hidruro de NADPH a FMN, generando FMNH2 y liberando NADP+. En la reacción oxidativa, una molécula organoarsénica se coloca cerca del 
FMNH2. La flavina reducida es oxidada por $\mathrm{O}_{2}$, produciendo $\mathrm{H}_{2} \mathrm{O}$, seguido de la oxidación del arsénico trivalente.

\section{DETERMINANTES DE RESISTENCIA MERCURIO Y SUS MECANISMOS}

En Bacillus thuringiensis PW-05 aislado de la costa de Odisha, se ha identificado el determinante merRTPA, siendo el conjunto de genes más simples para la resistencia al $\mathrm{Hg}^{2+}(30)$. El operón mer es regulado por el gen merR, sin embargo, en los Bacteroidetes aislados del Alto Ártico, no está presente este gen, sino ars $R$, en consecuencia, es posible que merR sea un desarrollo posterior en la evolución del operón mer, que remplaza a los genes reguladores ars $R$ y genera sistemas mer más eficientes (31). La proteína MerR en ausencia de $\mathrm{Hg}^{2+}$, reprime la transcripción de los genes estructurales al capturar la ARN polimerasa en un estado inactivo cerrado. En presencia de $\mathrm{Hg}^{2+}$, MerR se une a una secuencia de ADN en las regiones -35 y -10 de su ADN promotor. Tras la unión de $\mathrm{Hg}^{2+}$, los cambios de conformación de MerR inducen un subenrollamiento del ADN promotor, facilitando la formación de un complejo abierto para iniciar la transcripción (32).

Los genes merP y merT se distribuyen por todo el árbol filogenético (33). Por el contrario, Jan et al. (34) identificaron que de 18 cepas aisladas de sitios contaminados de la India, 14 aislados portaban el gen merPy solo 12 cepas el gen merT. Principalmente, P. aeruginosa ARY1 y Klebsiella sp. ND3 portaban el gen merP y merT. Además, Klebsiella pneumonia ND6 portaba el gen merP, pero carecía de merT, por lo tanto, se evidencia que la función de merT es compensada por otro gen transportador. El gen merP codifica para la proteína MerP, que elimina el $\mathrm{Hg}^{2+}$ o el $\mathrm{CH}_{3} \mathrm{Hg}$ en el periplasma y lo transfiere a los diferentes transportadores $(35,36)$. En el transposon Tn501 de $P$. aeruginosa, el $\mathrm{Hg}^{2+}$ es secuestrado por el par de grupos tiol en MerP y posteriormente, transferido al par de grupos tiol ubicados en el primer dominio transmembrana de MerT. A continuación, el $\mathrm{Hg}^{2+}$ unido, se pasa a través de la membrana hasta el par de cisteínas en la cara citoplásmica de MerT (37). Desde MerT, el $\mathrm{Hg}^{2+}$ pasa a MerA.
El gen merA es el determinante clave para la resistencia al $\mathrm{Hg}^{2+}$ y se encuentra omnimpresente en el entorno $(38,39)$. Sin embargo, Boyd y Barkay (33) establecen que el gen merA, no se encuentra en taxones y gremios microbianos completos y rara vez es identificado en anaerobios estrictos (33). En contraste, la bacteria aeróbica $P$. pseudoalcaligenes carece de merA, por lo tanto, la resistencia al $\mathrm{Hg}^{2+}$ depende de una estrategia mediada por merT y merP (40). Lian et al. (41) proponen un mecanismo para el gen merA presente en el Tn 501 de $P$. aeruginosa: Cis 11 y 14 están presentes en el dominio N- terminal de MerA; NmerA se une y entrega el $\mathrm{Hg}^{2+}$ al par de cisteínas C-terminal (Cis 558 y 559) (42). Después de que el $\mathrm{Hg}^{2+}$ se une a este par de cisteínas, la cola C-terminal cambia de conformación y mueve el complejo al interior de la proteína donde el $\mathrm{Hg}^{2+}$ se transfiere al par de cisteínas del sitio activo, Cis 136 y 141. El otro sustrato (NADPH), transfiere un hidruro a FAD, produciendo $\mathrm{FADH}_{2}$ y NADP+ oxidado. Luego, $\mathrm{FADH}_{2}$ reduce el complejo Cis141-S- $\mathrm{Hg}^{2+}-\mathrm{S}$ Cis136 para producir $\mathrm{Hg}^{0}$.

El gen merB del sistema de espectro ancho otorga la resistencia a los compuestos organomercuriales. Mindlin et al. (28) identifican el gen merB en la cepa $A$. Iwoffii ED23-35 aislada del permafrost. De modo similar, en el Tn 6294 de Bacillus sp. EOA1 aislada de Taiwan y Pseudomonas putida W619 aislada de un suelo contaminado con níquel está presente el gen merB $(43,44)$. Boyd y Barkay (33) han identificado que 42 cepas, principalmente, distribuidas entre los Bacteroidetes, Actinobacteria, Firmicutes y Proteobacterias portan el gen merB en su genoma. Paralelamente, se ha identificado la presencia del gen merB en las Gamma Proteobacterias aisladas del Alto Ártico (31). A partir de estos ejemplos, resulta evidente la distribución de merB en bacterias Gram negativas, sin embargo, se reporta que es más común en bacterias Gram positivas (45).

El producto génico de merB del plásmido $\mathrm{R} 831 \mathrm{~b}$ presenta la Cis96, 159 y Asp 99 que son los residuos catalíticos conservados en las proteínas MerB. Guo et al. (46) definen dos mecanismos llevados a cabo por merB. En el primero, el $\mathrm{CH}_{3}-$ $\mathrm{Hg}$ se une a uno de los dos residuos de cisteína 
del sitio activo (Cis96 o 159) (47). La otra cisteína dona un protón al grupo saliente $\left(\mathrm{CH}_{3}^{-}\right)$, generando la escisión del enlace y la formación de $\mathrm{CH}_{4}$. En el segundo mecanismo, el $\mathrm{CH}_{3}-\mathrm{Hg}$ se une a una de las dos cisteínas del sitio activo, pero en lugar de protonar el grupo saliente, la otra cisteína transfiere un protón a Asp99. Este paso permite que ambas cisteínas se coordinen con el metilmercurio. Tras la coordinación, Asp 99 protona el $\left(\mathrm{CH}_{3}^{-}\right)$saliente y produce los productos de ruptura $\mathrm{Hg}^{2+} \mathrm{y} \mathrm{CH}_{4}$.

Los operones mer han aumentado sus complementos genéticos y por tal razón su diversidad funcional (48). Los genes que codifican transportadores alternativos son comunes en operones de evolución reciente (33). Se ha identificado que el gen merG está presente (de 272 aislados) en 3 cepas de Pseudomona sp., y en 2 cepas aisladas del suelo, lo que sugiere la presencia de $\operatorname{mer} G$, solo en cepas que contengan el gen merB (33). El gen merF en Proteus mirabilis $\mathrm{PW} 4 \mathrm{c}$ ha sido identificado como un transportador, en cambio, en Pseudomonas stutzeri 273 aislada de sedimentos de mar, merF es el gen clave para la formación de flagelos (49, 48). Hwang et al. (50) caracterizaron la dinámica de MerF unido al $\mathrm{Hg}^{2+}$, al recibir el $\mathrm{Hg}^{2+}$ de MerP, la Cis 21 y 22 de MerF lo transfiere a Cis 71 y 72, las cuales lo transfieren a MerA en el citoplasma. MerF presenta dos pares de cisteínas universalmente conservadas, debido a su función en el transporte de $\mathrm{Hg}^{2+}$ (35).

\section{Determinantes de resistencia al cromato y sus mecanismos}

El determinante de resistencia al cromato (chr), se ha detectado en varios géneros bacterianos. Adekanmbi et al. (49) identificaron (de 40 cepas aisladas de aguas residuales de impresión) los genes chrAB en $P$. aeruginosa, Providencia vermicola PWAP3 y $P$. mirabilis PW4c corroborando la distribución de los genes chr en los plásmidos de bacterias Gram negativas. De manera similar, este mismo sistema de genes se ha identificado en los plásmidos de 40 aislados de las Enterobacteriaceae (de 109 aislados nosocomicales) y en las cepas ED9-5a y EK30A de A. Iwoffii $(51,28)$. En contraste, el gen chrA puede estar presente en plásmidos y cromosomas de Proteobacterias (Shewanella oneidensis MR1), Cyanobacteria, Actinobacteria y Firmicutes $(52,51,53)$.

O. tritici 5bvl1 y Burkholderia xenovorans LB400 contienen el determinante chrBACF ubicados en el cromosoma y en un mega plásmido respectivamente $(54,55)$. Cupriavidus metallidurans $\mathrm{CH} 34$ contiene genes para la resistencia al $\mathrm{Cr}^{6+}$ en el plásmido pMOL28 y en el cromosoma (56). No obstante, solamente $c h r B$ y chrA son esenciales para la resistencia al cromato (57). Tanto en O. tritici 5bvl1 y Lysinibacillus sphaericus Ot4b.31; ChrB se induce por $\mathrm{Cr}^{6+}$, esta interacción evita que ChrB se una a la región promotora generando una desrepresión del sistema que conduce a la expresión de los genes estructurales $(54,58)$. En contraste, ChrB de C. metallidurans $\mathrm{CH} 34$ activa el sistema chr en respuesta al cromato y sulfato (54). De manera similar, Verduzo y Julia (59) establecen que ChrS de Bacillus subtilis 168, regula negativamente el operón chr por la unión de ChrS a la región reguladora de su propio gen, y lo regula positivamente por $\mathrm{Cr}^{6+}(59)$.

Branco y Morais (61) establecen la importancia de los genes $c h r C$ y $c h r F$ para evitar la acumulación de especies reactivas de oxígeno (ROS) en las células sometidas a estrés por cromato; además, establecen una gran similitud entre el producto génico de $c h r C$ de la cepa 5 bvll con la Fe-SOD (ChrC) de C. metallidurans $\mathrm{CH} 34$. En contraste, el producto génico de $c h r F$ de Cupriavidus neocaledonicus STM 6070 aislada de un suelo rico en níquel y el producto del gen $c h r I$ de $C$. metallidurans $\mathrm{CH} 34$ han sido identificados como represores del operón chr $(62,63)$. Sin embargo, esto difiere de otro estudio, en el cual identifican que $c h r F$ codifica para un superóxido dismutasa (Mn-SOD) (61).

Determinantes de resistencia al cadmio y sus mecanismos

El sistema de resistencia al cadmio en las bacterias Gram positivas es el sistema cad, se ha identificado el conjunto de genes cadCA en S. aureus ATCC12600 aislado de un ambiente 
hospitalario, en Bacillus vietamensis 151-6 y Bacillus marisflavi 151-25 aisladas de un suelo contaminado con cadmio $(64,65,66,67)$. Por otro lado, en el genoma de la cepa JMAK1 de Bacillaceae aislada de la estación Concordia de la Antártica, se ha evidenciado la presencia de los genes $c a d A, c a d C$ y cadD (68). El gen $c a d C$ codifica para el represor CadC que se disocia del operador/promotor de cad tras la unión de $\mathrm{Cd}^{2+}$ (65). CadC de la cepa ATCC12600 presenta dos sitios de unión al metal (64). Por el contrario, CadC de Listeria monocytogenes EGDe, carece del sitio de tipo II. En esta cepa, CadC es un regulador dependiente de $\mathrm{Cd}^{2+}$ para la expresión de $c a d A C$ y $l s p B$ (69).

Los genes cadXD son otro mecanismo de resistencia al cadmio y zinc; se ha identificado este sistema en S. aureus ST398-t571 aislado de una muestra nasal (70). Del mismo modo, se ha encontrado en $S$. aureus y A. ferrooxidans ATCC 23270, el operón cadXB y cadAB respectivamente $(71,72)$. Adicionalmente, la resistencia al cadmio en $P$. aeruginosa $\mathrm{BC} 15$ aislada de aguas residuales y $P$. putida W619 aislada de un sitio contaminado con níquel; se logra mediante la función de dos genes vitales $c a d R$ y $\operatorname{cadA}(73,74)$.

CadR de $P$. aeruginosa $\mathrm{BC} 15$, reprime su propia expresión y la de cadA en ausencia de $\mathrm{Cd}^{2+}$, pero se induce en su presencia. CadR presenta 2 tipos distintos de sitios de unión a metales en el extremo C-terminal. Prabhakaran et al. (74) establecen que los residuos de cisteína inician la expresión de CadR desde su promotor, además, la curvatura en PcadR permite a la ARN polimerasa acceder al sitio completo del promotor para la transcripción. Al unirse el $\mathrm{Cd}^{2+}$ al sitio I, orienta el dominio C-terminal, cambiando el promotor de un estado represor a un estado parcialmente distorsionado. Con una mayor unión de $\mathrm{Cd}^{2+}$ al sitio II, CadR se estabiliza y se transforma en un activador estable (23)

Tynecka et al. (82) proponen un mecanismo llevado a cabo por CadA para extruir el $\mathrm{Cd}^{2+}$ : dos $\mathrm{Cd}^{2+}$ ingresan a través del uniportador de $\mathrm{Mn}^{2+}$, la unión de $\mathrm{Cd}^{2+}$ a CadA provoca la fosforilación de la proteína por ATP, y un cambio de conformación de la cara citoplasmática al periplasma. En consecuencia, un mayor número de protones bombeados a través de la cadena respiratoria compiten con el $\mathrm{Cd}^{2+}$ externo y se unen a sitios de superficie de baja afinidad en CadA. Finalmente, los protones cotransportados hacia abajo a través del canal, extruden dos $\mathrm{Cd}^{2+}$ a través del intercambio $\mathrm{Cd}^{2+} / \mathrm{H}^{+}$.

Un sistema de resistencia al $\mathrm{Cd}^{2+}, \mathrm{Co}^{2+}$ y $\mathrm{Zn}^{2+}$ en bacterias Gram negativas es el sistema $(c z c)$. P. putida SB32 y Pseudomonas monteilii SB35 contienen en sus plásmidos el sistema $c z c$ $(65,75)$. En A. ferrooxidans ATCC 23270 y en Marinobacter adhaerens HP15 aislada de un entorno marino, se han encontrado los genes: $c z c A, c z c B$ y $c z c C$, además, se ha evidenciado que la bomba $c z c A B C$ en la cepa HP15 es específica para zinc $(72,76,77)$. El sistema $c z c I C B A$ es el determinante más conservado en el género Cupriavidus, por lo tanto, resulta evidente su presencia en $C$. metallidurans $\mathrm{CH} 34$ y BS1 $(78,77)$.

\section{Potencial biotecnológico de los determinantes genéticos}

La constante búsqueda de nuevas cepas microbianas potencialmente resistentes a iones tóxicos, ha abierto el camino hacia el descubrimiento de tecnologías innovadoras basadas en el empleo de estos microorganismos. Es así que los determinantes de resistencia poseen una gama de aplicaciones, entre estas se incluyen su uso para la construcción de biosensores y de organismos útiles para la descontaminación del medio $(79,86,83)$.

La tabla 1, detalla los avances más actuales en el desarrollo de biosensores mediante el uso de genes reguladores: $\operatorname{ars} R, \operatorname{mer} R, \mathrm{cad} C$, y $\mathrm{chr} B$

\begin{tabular}{|l|l|l|l|}
\hline \multicolumn{1}{|c|}{ Biosensor } & $\begin{array}{c}\text { Rango de } \\
\text { detección }\end{array}$ & $\begin{array}{c}\text { Gen } \\
\text { bacteriano }\end{array}$ & Referencia \\
\hline $\begin{array}{l}\text { Biorreportador bioluminis- } \\
\text { cente para la evaluación de } \\
\text { la contaminación por arséni- } \\
\text { co en muestras de agua de la } \\
\text { India }\end{array}$ & $0,74-60$ & & \\
$\mu \mathrm{L} \mathrm{As}{ }^{3+}$ & & $\operatorname{arsR-lux}$ & $(79)$ \\
\hline $\begin{array}{l}\text { Biosensor bacteriano a base } \\
\text { de color de bajo costo para } \\
\text { medir el arsénico en el agua } \\
\text { subterránea. }\end{array}$ & $\mu \mathrm{g} / \mathrm{L} \mathrm{As} \mathrm{s}^{3+}$ & & \\
\hline
\end{tabular}




\begin{tabular}{|c|c|c|c|}
\hline $\begin{array}{l}\text { Biosensor bacteriano para la } \\
\text { detección de mercurio en so- } \\
\text { luciones liquidas. }\end{array}$ & $\begin{array}{l}10^{-4}{\mathrm{y} 10^{-8}}^{\mathrm{M} \mathrm{Hg}^{2+}}\end{array}$ & merR-gfp & (81) \\
\hline $\begin{array}{l}\text { Biosensor bacteriano de cé- } \\
\text { lulas completas para la detec- } \\
\text { ción de cadmio. }\end{array}$ & $\begin{array}{l}1 \mathrm{mM} \\
1 \mu \mathrm{M} \\
10 \mathrm{nM} \\
\mathrm{Cd}^{2+} \\
\end{array}$ & cadC-gfp & (79) \\
\hline $\begin{array}{l}\text { Bioinformadores de células } \\
\text { enteras altamente sensibles y } \\
\text { específicos para la detección } \\
\text { de cromato en muestras am- } \\
\text { bientales }\end{array}$ & $\begin{array}{l}1 \mu \mathrm{M}-50 \\
\mu \mathrm{M} \mathrm{Cr}{ }^{6+} \\
0,1->50 \\
\mu \mathrm{M} \mathrm{Cr} r^{6+}\end{array}$ & $c h r B-g f p$ & (83) \\
\hline
\end{tabular}

Tabla 1. Biosensores basados en diferentes genes de resistencia a metales pesados

Una mayor familiaridad con el circuito regulador de los determinantes genéticos se ha aprovechado para la construcción de biosensores. Sharma et al. (79) altera E. coli DH5a para producir luz cuando entra en contacto con iones de $\mathrm{As}^{3+}$. Fusionaron el gen arsR y el gen lux. El biosensor exhibió un rango de detección de 0,74 a 60 $\mu \mathrm{g} / \mathrm{L} \mathrm{As}^{3+}$, la luz fue emitida a los 30 minutos de exposición, obteniendo resultados confiables a las 2 h. En contraste, Huang et al. (80) generaron un biosensor basado en color; el casete arsR-lac $Z$ fue insertado en la cepa de E. coli DH5. Su rango de detección fue de 10 a $500 \mu \mathrm{g} / \mathrm{L}$ de $\mathrm{As}^{3+}$ y a las $3 \mathrm{~h}$ se evidenció el color azul. Este biosensor provee una señal de color confiable, y es útil para la detección rápida del $\mathrm{As}^{3+}$.

Debido a la alta toxicidad del mercurio en el agua, Roointan et al. (81) han construido un biosensor usando el gen merR de Pseudomonas sp. y la proteína de fluorescencia verde (GFP) en la cepa E. coli BL21 (DE3). Su rango de detección fue de $10^{-4}$ y $10^{-8} \mathrm{M}$ a las $3 \mathrm{~h}$. A concentraciones bajas la expresión de GFP es baja y a concentraciones altas la expresión de GFP se reduce. La sensibilidad y el breve tiempo de respuesta resulta ventajoso para la detección in situ de la contaminación por mercurio en agua. Según los valores estándar de mercurio en agua $(0,001 \mathrm{mg} / \mathrm{L})$, este rango de detección es consistente para su aplicación. Sin embargo, debido a la falta de merA en el biosensor, se dio una reducción de la intensidad de la fluorescencia a altas concentraciones.

Por otro lado, el gen regulador del operón cad se usó para la construcción de un biosensor microbiano en respuesta a $\mathrm{Cd}^{2+}$; E. coli BL21 (DE3) se empleó como una cepa bacteriana del sensor; el promotor/operador de cad, el gen $c a d C$ y $g f p$. La concentración detectable más baja del biosensor a $37^{\circ} \mathrm{C}$ fue $1 \mathrm{mmol} / \mathrm{L}$, disminuyendo a $1 \mu \mathrm{mol} / \mathrm{L}$ a $25{ }^{\circ} \mathrm{C}$ y $10 \mathrm{nmol} / \mathrm{L}$ a $15{ }^{\circ} \mathrm{C}$. Una de las ventajas de este biosensor es la mejora de la sensibilidad a baja temperatura, así como la respuesta selectiva y dependiente de la dosis de $\mathrm{Cd}^{2+}$.

Paralelamente, el desarrollo de bioinformadores para la detección de cromato, se basan en la expresión de $g f p$ bajo el control del promotor $c h r$ y el gen regulador chrB. E. coli y $O$. tritici fueron diseñados para emitir fluorescencia en presencia de $\mathrm{Cr}^{6+}$. El biosensor de E. coli demostró ser específico y sensible, su rango de detección fue de $0,1 \mu \mathrm{M}$ a $50 \mu \mathrm{M}$ y la máxima fluorescencia se alcanzó a las 5 horas. En $O$. tritici el rango de detección fue de $1 \mu \mathrm{M}$ a $>50 \mu \mathrm{M}$, en este rango se dio la saturación de la señal de fluorescencia. Los 2 biosensores resultaron ser específicos y proporcionan utilidad en campo (83).

La tabla 2 detalla los microorganismos o plantas transgénicas que expresan determinantes de resistencia.

\begin{tabular}{|l|l|l|c|}
\hline $\begin{array}{l}\text { Planta o } \\
\text { microorganismos } \\
\text { modificado }\end{array}$ & $\begin{array}{l}\text { Gen } \\
\text { bacteriano }\end{array}$ & Nivel de resistencia & Referencia \\
\hline $\begin{array}{l}\text { P. putida KT2440 } \\
\text { Escherichia coli BL21 } \\
\text { (DE3). }\end{array}$ & ars $M$ & $10 \mathrm{mM} \mathrm{As}^{3+}$ & $(72)$ \\
\hline $\begin{array}{l}\text { A. thaliana } \\
\text { A. thaliana y }\end{array}$ & $m e r T$ & $100 \mu \mathrm{M} \mathrm{Cr}^{6+}$ & $(85)$ \\
\hline $\begin{array}{l}\text { Nicotiana. tabacum } \\
\text { Oryza sativa }\end{array}$ & $m e r A B$ & $\begin{array}{l}10,000 \mu \mathrm{mg} / \mathrm{kg} \mathrm{HgCl}_{2} \\
100 \mu \mathrm{g} / \mathrm{kg} \mathrm{CH} \mathrm{CgCl}_{3}\end{array}$ & $(84)$ \\
\hline $\begin{array}{l}\text { Solanum } \\
\text { lycopersicum }\end{array}$ & $m e r A B$ & $\begin{array}{l}4000 \mu \mathrm{g} / \mathrm{kg} \mathrm{HgCl}_{2} \\
4000 \mu \mathrm{g} / \mathrm{kg} \mathrm{CH}_{3} \mathrm{HgCl}\end{array}$ & $(84)$ \\
\hline
\end{tabular}

Tabla 2. Plantas y bacterias que expresan genes de resistencia a metales

La comprensión de los determinantes genéticos $\mathrm{y}$ las funciones que codifican ha facilitado la bioingeniería de células y plantas (33). Chen et al. (72) diseñaron genéticamente $P$. putida KT2440 con expresión estable de arsM-gfp. La cepa creció a concentraciones de $\mathrm{As}^{3+}$ de hasta 10 $\mathrm{mM}$ a diferencia de los controles, además, mostró una alta actividad de metilación y volatilización cuando se expuso a $\mathrm{As}^{3+} \mathrm{o} \mathrm{As}^{5+} 25 \mu \mathrm{M}$, después de $48 \mathrm{~h}$, la mayor parte del arsénico fue metilado al ácido dimetilarsínico $\left(\mathrm{DMA}^{5+}\right)$, ácido monometilarsónico $\left(\mathrm{MA}^{5+}\right)$ y gas trimetilarsina $\left(\mathrm{TMA}^{5+}\right)$. Esta capacidad de resistir al $\mathrm{As}^{3+}$, la hace una candidata para la remediación in situ 
de suelos contaminados.

Zhou et al. (85) construyeron cepas de E. coli BL21 (DE3) que expresan el gen chrB de O. tritici 5 bvl1. La expresión de $c h r B$ la realizaron intracelular y en superficie y compararon la capacidad de adsorción de $\mathrm{Cr}^{6+}$. Un nivel bajo de $\mathrm{Cr}^{6+}(<10 \mu \mathrm{M})$ en el medio, no inhibió el crecimiento de ninguna de las cepas. A concentraciones mayores a 100 $\mu \mathrm{M} \mathrm{Cr}^{6+}$, las células que expresan ChrB eran más resistentes a la toxicidad que las células control. ChrB expuesto en superficie eliminó el 99,1\% del $\mathrm{Cr}^{6+}$ total (concentración inicial 0,5 mM) en $2 \mathrm{~h}$. La capacidad máxima de adsorción de células de E. coli BL21 que expresan chrB intracelular fue de $235 \mu \mathrm{mol} / \mathrm{g}$ de peso seco de $\mathrm{Cr}^{6+}$.

$\mathrm{Xu}$ et al. (86) generaron plantas de A. thaliana que expresan el gen merT de Pseudomonas alcaligenes. A. thaliana mejora la formación de la raíz bajo $10 \mathrm{mmol} / \mathrm{L}$ de $\mathrm{HgCl}_{2}$; después de 5 días de estrés los controles mostraron disminución del contenido de clorofila, en contraste con las plantas que expresan merT (18,65\% y $22,43 \%)$. Se evidenció menor contenido de sustancias reactivas al ácido tiobarbitúrico (TBARS) y de ROS.

Las plantas no poseen la capacidad de desintoxicar el $\mathrm{CH}_{3} \mathrm{Hg}$, por tal razón, varias investigaciones se han enfocado en generar plantas que expresen el gen merB confiriéndoles la capacidad de desintoxicar por sí mismas este elemento (60). Li et al. (84) expresaron los genes mer $A B$ en $A$. thaliana, $N$. tabacum, $O$. satyva y $S$. lycopersicum. Demostraron que $N$. tabacum y $A$. thaliana transgénicas muestran resistencia a $10.000 \mu \mathrm{g} / \mathrm{kg}$ de $\mathrm{HgCl}_{2}$ y $100 \mu \mathrm{g} / \mathrm{kg}$ de $\mathrm{CH}_{3} \mathrm{HgCl}$. El crecimiento de S. lycopersicum y O. satyva se inhibió a $4000 \mu \mathrm{g} / \mathrm{kg}$ de mercurio. Las plantas transgénicas redujeron 4 veces más la concentración de mercurio a diferencia de los controles. La expresión de estos genes permite la construcción de plantas útiles para procesos de fitorremediación.

\section{CONCLUSIONES}

Los determinantes genéticos de resistencia a metales pesados principalmente para el arsénico, mercurio, cromo y cadmio son ars, mer, chr, $c a d, c z c$ respectivamente. Estos se encuentran distribuidos en el genoma de una amplia gama de bacterias aisladas de entornos altamente contaminados; en estos sitios se ha identificado genes de resistencia que muestran una gran especificidad para los iones metálicos o sus derivados orgánicos.

A lo largo de la revisión se ha resaltado los mecanismos de acción llevados a cabo por los genes reguladores como: arsR, merR, $\operatorname{chr} B$, cadC, cadR; del mismo modo, el mecanismo de reducción, metilación, desmetilación, y oxidación de los iones de metales pesados llevados a cabo por merA, merB, ars A, arsI, $\operatorname{ars} M$ y $\operatorname{arsH}$. Existen bombas de eflujo para el mecanismo de expulsión de los metales cuando ingresan a la célula tal es el caso de $\operatorname{ch} r A, \operatorname{cad} A$ respectivamente para el cromato y el cadmio. En el caso del mercurio hay una variedad de genes cuyo mecanismo es el transporte del metal en su estado orgánico e inorgánico merE, merT, merF, merC cumplen esta función, del mismo modo, para el arsénico existen dos determinantes para el transporte $\operatorname{ars} B$ y $a c r 3$.

Dentro de las aplicaciones de los determinantes genéticos se encuentran su uso para construcción de biosensores mediante la fusión de los genes reguladores como arsR, merR, cadC, chrB del operón y genes reporteros como gfp o lux para la detección de metales pesados, siendo un método económico y eficaz en comparación con los métodos tradicionales. Otra de las posibles aplicaciones que se le otorga a los determinantes de resistencia es en la generación de organismos transgénicos que expresen genes de interés y que puedan ser usados para la remediación de la contaminación. 
eferencias

1. Kruger MC, Bertin PN, Heipieper HJ, Arsène-Ploetze F. Bacterial metabolism of environmental arsenic - Mechanisms and biotechnological applications. Appl Microbiol Biotechnol. 2013;97(9):3827-41.

2. Ordoñez OF, Lanzarotti E, Kurth D, Cortez N. Genome comparison of two Exiguobacterium strains from high altitude andean lakes with different arsenic resistance: identification and 3D modeling of the Acr3 efflux pump. Front Environ Sci. 2015;3(July):1-12.

3. Andres J, Bertin PN. The microbial genomics of arsenic. FEMS Microbiol Rev. 2016;40(2):299-322.

4. Fekih I Ben, Zhang C, Li YP, Zhao Y, Alwathnani HA, Saquib Q, et al. Distribution of arsenic resistance genes in prokaryotes. Front Microbiol [Internet]. 2018;9(OCT):1-11. Available from: https://www.ncbi.nlm.nih.gov/pmc/articles/PMC6205960/

5. Uhrynowski W, Radlinska M, Drewniak L. Genomic analysis of Shewanella sp. O23s-the natural host of the psheb plasmid carrying genes for arsenic resistance and dissimilatory reduction. Int J Mol Sci. 2019;20(5).

6. Antonucci I, Gallo G, Limauro D, Contursi P, Ribeiro AL, Blesa A, et al. An ArsR/SmtB family member regulates arsenic resistance genes unusually arranged in Thermus thermophilus HB27. Microb Biotechnol. 2017;10(6):1690-701.

7. Shen Z, Han J, Wang Y, Sahin O, Zhang Q. The Contribution of ArsB to Arsenic Resistance in Campylobacter jejuni. PLoS One. 2013;8(3):1-8.

8. Zhao C, Zhang Y, Chan Z, Chen S, Yang S. Insights into arsenic multi-operons expression and resistance mechanisms in Rhodopseudomonas palustris CGA009. Front Microbiol. 2015;6(SEP):1-8.

9. Villadangos AF, Van Belle K, Wahni K, Tamu Dufe V, Freitas S, Nur H, et al. Corynebacterium glutamicum survives arsenic stress with arsenate reductases coupled to two distinct redox mechanisms. Mol Microbiol. 2011;82(4):998-1014.

10. Xiao KQ, Li LG, Ma LP, Zhang SY, Bao P, Zhang T, et al. Metagenomic analysis revealed highly diverse microbial arsenic metabolism genes in paddy soils with low-arsenic contents. Environ Pollut [Internet]. 2016;211:1-8. Available from: http://dx.doi.org/10.1016/j.envpol.2015.12.023

11. Suhadolnik MLS, Costa PS, Castro GM, Lobo FP, Nascimento AMA. Comprehensive insights into arsenic- and iron-redox genes, their taxonomy and associated environmental drivers deciphered by a meta-analysis. Environ Int. 2021;146(106234):1-11.

12. Prieto-Barajas CM, Elorza-Gómez JC, Loeza-Lara PD, Sánchez-Yáñez JM, Valencia-Cantero E, Santoyo G. Identificación y análisis de genes ars en cepas de Bacillus hipertolerantes al arsénico, aisladas de pozas termales en Araró, México. TIP Rev Espec en Ciencias Químico-Biológicas [Internet]. 2018;21(Iii):22-9. Available from: http://creativecommons.org/licenses/by-nc-nd/4.0/) 13. Li X, Zhang L, Wang G. Genomic evidence reveals the extreme diversity and wide distribution of the arsenic-related genes in Burkholderiales. PLoS One. 2014;9(3):1-11.

14. Jia MR, Tang N, Cao Y, Chen Y, Han YH, Ma LQ. Efficient arsenate reduction by As-resistant bacterium Bacillus sp. strain PVR-YHB1-1: Characterization and genome analysis. Chemosphere [Internet]. 2019;218:1061-70. Available from: https://doi.org/10.1016/j.chemosphere.2018.11.145 15. Sousa T, Branco R, Piedade AP, Morais P V. Hyper accumulation of arsenic in Mutants of Ochrobactrum tritici silenced for arsenite efflux pumps. 2015;1-14.

16. Shen Z, Luangtongkum T, Qiang Z, Jeon B, Wang L, Zhang Q. Identification of a novel membrane transporter mediating resistance to organic arsenic in Campylobacter jejuni. Antimicrob Agents Chemother. 2014;58(4):2021-9.

17. Jiang H, Liang Y, Yin H, Xiao Y, Guo X, Xu Y, et al. Effects of arsenite resistance on the growth and functional gene expression of Leptospirillum ferriphilum and Acidithiobacillus thiooxidans in pure culture and coculture. Biomed Res Int. 2015;2015:1-13.

18. Yang HC, Rosen BP. New mechanisms of bacterial arsenic resistance. Biomed J [Internet]. 
2016;39(1):5-13. Available from: http://dx.doi.org/10.1016/j.bj.2015.08.003

19. Villadangos AF, Fu HL, Gil JA, Messens J, Rosen BP, Mateos LM. Efflux permease CgAcr3-1 of Corynebacterium glutamicum is an arsenite-specific antiporter. J Biol Chem [Internet]. 2012;287(1):723-35. Available from: http://dx.doi.org/10.1074/jbc.M111.263335

20. Yu X, Zheng W, Bhat S, Aquilina JA, Zhang R. Transcriptional and posttranscriptional regulation of Bacillus sp. CDB3 arsenic-resistance operon ars1. PeerJ [Internet]. 2015;2015(9):1-15. Available from: https://peerj.com/articles/1230/

21. Yoshinaga $\mathrm{M}$, Rosen BP. A C.As lyase for degradation of environmental organoarsenical herbicides and animal husbandry growth promoters. Proc Natl Acad Sci USA [Internet]. 2014;111(21):7701-6. Available from: www.pnas.org/lookup/suppl/doi:10. 1073/ pnas.1403057111/-/DCSupplemental.

22. Yang J, Abdul Salam AA, Rosen BP. Genetic mapping of the interface between the ArsD metallochaperone and the ArsA ATPase. Mol Microbiol [Internet]. 2011;79(4):872-81. Available from: https://onlinelibrary.wiley.com/doi/full/10.1111/j.1365-2958.2010.07494.x

23. Liu X, Hu Q, Yang J, Huang S, Wei T, Chen W, et al. Selective cadmium regulation mediated by a cooperative binding mechanism in CadR. Proc Natl Acad Sci U S A. 2019;116(41):20398-403. 24. Firrincieli A, Presentato A, Favoino G, Marabottini R, Allevato E, Stazi SR, et al. Identification of resistance genes and response to arsenic in Rhodococcus aetherivorans BCP1. Front Microbiol. 2019;10(MAY):1-13.

25. Nadar VS, Yoshinaga M, Pawitwar SS, Kandavelu P, Sankaran B, Rosen BP. Structure of the ArsI C-As Lyase: Insights into the mechanism of degradation of organoarsenical herbicides and growth promoters. J Mol Biol [Internet]. 2016;428(11):1-32. Available from: http://dx.doi. org/10.1016/j.jmb.2016.04.022

26. Chen J, Nadar VS, Rosen BP. A novel MAs(III)-selective ArsR transcriptional repressor. Mol Microbiol. 2017;106(3):469-78.

27. Páez-Espino AD, Nikel PI, Chavarría M, Lorenzo V De, Rica C, José S, et al. ArsH protects Pseudomonas putida from oxidative damage caused by exposure to arsenic. Environ Microbiol. 2020;22:2230-42.

28. Mindlin S, Petrenko A, Kurakov A, Beletsky A, Mardanov A, Petrova M. Resistance of Permafrost and Modern Acinetobacter lwoffii Strains to Heavy Metals and Arsenic Revealed by Genome Analysis. Biomed Res Int. 2016;2016:1-10.

29. Chen J, Bhattacharjee H, Rosen BP. ArsH is an organoarsenical oxidase that confers resistance to trivalent forms of the herbicide monosodium methylarsenate and the poultry growth promoter roxarsone. Mol Microbiol. 2015;96(5):1042-52.

30. Dash HR, Mangwani N, Das S. Characterization and potential application in mercury bioremediation of highly mercury-resistant marine bacterium Bacillus thuringiensis PW-05. Investig en ciencias Ambient y Contam. 2013;1-12.

31. Møller AK, Barkay T, Hansen MA, Norman A, Hansen LH, Sørensen SJ, et al. Mercuric reductase genes (merA) and mercury resistance plasmids in High Arctic snow, freshwater and seaice brine. FEMS Microbiol Ecol [Internet]. 2013;87:52-63. Available from: https://academic.oup. com/femsec/article/87/1/52/508980

32. Wang D, Huang S, Liu P, Liu X, He Y, Chen W, et al. Structural analysis of the $\mathrm{Hg}^{2+}$ regulatory protein Tn501 MerR from Pseudomonas aeruginosa. Nat Publ Gr. 2016;(August):1-9.

33. Boyd E, Barkay T. The mercury resistance operon: from an origin in a geothermal environment to an efficient detoxification machine. Front Microbiol. 2012;3(10):1-13.

34. Jan AT, Azam M, Choi I, Ali A, Haq QMR. Analysis for the presence of determinants involved in the transport of mercury across bacterial membrane from polluted water bodies of India. Brazilian J Microbiol [Internet]. 2016;47(1):55-62. Available from: http://dx.doi.org/10.1016/j. bjm.2015.11.023

35. Sone Y, Nakamura R, Pan-hou H, Itoh T, Kiyono M. Role of MerC, MerE, MerF, MerT, 
and/or MerP in resistance to mercurials and the transport of mercurials in Escherichia coli. Biol Pharm Bull. 2013;36(November):1835-41.

36. Sone Y, Uraguchi S, Takanezawa Y, Nakamura R, Pan-hou H, Kiyono M. Cysteine and histidine residues are involved in Escherichia coli Tn 21 MerE methylmercury transport. Front Microbiol. 2017;7:1994-9.

37. Ly K, Ryan LO, Mitra AK. Overexpression, purification and biophysical characterisation of Escherichia coli MerT. PROTEIN Expr Purif [Internet]. 2014;(December):1-5. Available from: http://dx.doi.org/10.1016/j.pep.2014.11.016

38. Ruuskanen MO, Poulain AJ. Swift evolutionary response of microbes to a rise in anthropogenic mercury in the Northern Hemisphere. ISME J [Internet]. 2020;(14:788-800):788-800. Available from: http://dx.doi.org/10.1038/s41396-019-0563-0

39. Chenia HY, Jacobs A. Antimicrobial resistance, heavy metal resistance and integron content in bacteria isolated from a South African tilapia aquaculture system. Dis Aquat Organ. 2017;126(3):199-209.

40. Zhang J, Zeng Y, Liu B, Deng X. MerP/MerT-mediated mechanism: A different approach to mercury resistance and bioaccumulation by marine bacteria. J Hazard Mater [Internet]. 2020;388(January):122062. Available from: https://doi.org/10.1016/j.jhazmat.2020.122062

41. Lian P, Guo H, Riccardi D, Dong A, Parks JM, Xu Q, et al. X-ray structure of a $\mathrm{Hg}^{2+}$ complex of mercuric reductase (MerA) and quantum mechanical/molecular mechanical study of $\mathrm{Hg}^{2+}$ transfer between the C-terminal and buried catalytic site cysteine pairs. Biochemistry. 2014;53(46):7211-22.

42. Johs A, Harwood IM, Parks JM, Nauss RE, Smith JC, Liang L, et al. Structural characterization of intramolecular $\mathrm{Hg}^{2+}$ transfer between flexibly linked domains of mercuric ion reductase. J Mol Biol [Internet]. 2011;413(3):639-56. Available from: http://dx.doi.org/10.1016/j. jmb.2011.08.042

43. Matsui K, Yoshinami S, Narita M, Chien M, Phung LT, Silver S, et al. Mercury resistance transposons in Bacillus strains from different geographical regions. 2016;(December 2015):1-8.

44. Taghavi S, Zhu W, Ramos J, Lelie D Van Der. Comparative genomics and functional analysis of niche-specie. FEMS Microbiol Rev [Internet]. 2011;35:299-323. Available from: https:// www.ncbi.nlm.nih.gov/pmc/articles/PMC3056050/

45. Naguib MM, El-Gendy AO, Khairalla AS. Microbial diversity of mer operon genes and their potential rules in mercury bioremediation and resistance. Open Biotechnol J. 2018;12(1):5677.

46. Guo H, Parks JM, Johs A, Smith JC. Mercury detoxification by bacteria: simulations of transcription activation and mercury - carbon bond cleavage. 2011;(Md):311-24.

47. Silva PJ, Rodrigues V. Mechanistic pathways of mercury removal fromthe organomercurial lyase active site. PeerJ. 2015;2015(7):1-18.

48. Zheng R, Wu S, Ma N, Sun C. Genetic and physiological adaptations of marine bacterium Pseudomonas stutzeri 273 to mercury stress. 2018;9(April):1-14.

49. Adekanmbi AO, Adelowo OO, Okoh AI, Fagade OE. Metal-resistance encoding gene-fingerprints in some bacteria isolated from wastewaters of selected printeries in Ibadan, South-western Nigeria. J Taibah Univ Sci. 2019;13(1):266-73.

50. Hwang H, Hazel A, Lian P, Smith JC, Gumbart JC, Parks JM. A Minimal membrane metal transport system: dynamics and energetics of mer proteins. J Comput Chem. 2020;41(6):528-37.

51. Caballero-Flores GG, Acosta-Navarrete YM, Ramírez-Díaz MI, Silva-Sánchez J, Cervantes C. Chromate-resistance genes in plasmids from antibiotic-resistant nosocomial enterobacterial isolates. FEMS Microbiol Lett. 2012;327(2):148-54.

52. Baaziz H, Gambari C, Boyeldieu A, Chaouche AA, Alatou R, Fons M. ChrA SO , the chromate efflux pump of Shewanella oneidensis, improves chromate survival and reduction. 2017;(Vi):1-15. 
53. Henson MW, Domingo JWS, Kourtev PS, Jensen R V., Dunn JA, Learman DR. Metabolic and genomic analysis elucidates strain-level variation in Microbacterium sp. isolated from chromate contaminated sediment. PeerJ. 2015;2015(11):1-17.

54. Morais Vasconcelos P, Branco R, Romeu F. Chromium resistance strategies and toxicity: what makes Ochrobactrum tritici 5bvl1 a strain highly resistant. Biometals (2011) [Internet]. 2011;24:401-10. Available from: https://link.springer.com/article/10.1007\%2Fs10534-011-9446-1

55. Acosta-Navarrete YM, León-Márquez YL, Salinas-Herrera K, Jácome-Galarza IE, Meza-Carmen V, Ramírez-Díaz MI, et al. Expression of the six chromate ion transporter homologues of Burkholderia xenovorans LB400. Microbiol (United Kingdom). 2014;160(PART 2):287-95.

56. Bazzis H. Isolation of Shewanella sp. from Algeria and characterization of a system involved in detoxification of chromate [Internet]. Freres Mentouri Cinstantine 1 University; 2018. Available from: http://archives.umc.edu.dz/handle/123456789/136458

57. Pradhan SK, Singh NR, Rath BP, Thatoi H. Bacterial chromate reduction: A review of important genomic, proteomic, and bioinformatic analysis. Crit Rev Environ Sci Technol. 2016;46(2122):1-82.

58. Rangel D, Dussan J. International Biodeterioration \& Biodegradation Transcriptional analysis and molecular dynamics simulations reveal the mechanism of toxic metals removal and e ffl ux pumps in Lysinibacillus. Int Biodeterior Biodegrad [Internet]. 2018;127(June 2017):46-61. Available from: https://doi.org/10.1016/j.ibiod.2017.11.016

59. Verduzco-rosas EASJLA, Julia A. An Lrp-type transcriptional regulator controls expression of the Bacillus subtilis chromate transporter. Antonie Van Leeuwenhoek. 2013;104 (941-948):1-8. 60. Paisio CE, González PS, Talano MA, Agostini E. Remediación biológica de Mercurio : Recientes avances Resumen Biological remediation of Mercury: Recent advances Abstract. 2012; 3(2):119-46.

61. Branco R, Morais P. Two superoxide dismutases from TnOtchr are involved in detoxification of reactive oxygen species induced by chromate. BMC Microbiol [Internet]. 2016;16(1):1-10. Available from: http://dx.doi.org/10.1186/s12866-016-0648-0

62. Klonowska A, Moulin L, Ardley JK, Braun F, Gollagher MM, Zandberg JD, et al. Novel heavy metal resistance gene clusters are present in the genome of Cupriavidus neocaledonicus STM 6070, a new species of Mimosa pudica microsymbiont isolated from heavy-metal-rich mining site soil. BMC Genomics. 2020;21(1):1-18.

63. Mergeay M, Houdt R Van. Metal response in Cupriavidus metallidurans. Biometals. 2015;I:1-97.

64. Hoogewerf AJ, Dyk LA Van, Buit TS, Roukema D, Resseguie E, Plaisier C, et al. Functional characterization of a cadmium resistance operon in Staphylococcus aureus ATCC12600: CadC does not function as a repressor. J Basic Microbiol. 2015;55:148-59.

65. Yu X, Ding Z, Ji Y, Zhao J, Liu X, Tian J, et al. An operon consisting of a p-type ATPase gene and a transcriptional regulator gene responsible for cadmium resistances in Bacillus vietamensis 151-6 and Bacillus marisflavi 151-25. BMC Microbiol. 2020;20(1):1-13.

66. Zhang H, Zhou Y, Bao H, Zhang L, Wang R, Zhou X. Plasmid-borne cadmium resistant determinants are associated with the susceptibility of Listeria monocytogenes to bacteriophage. Microbiol Res [Internet]. 2015;172:1-6. Available from: http://dx.doi.org/10.1016/j.micres.2015.01.008 67. Parsons C, Lee S, Kathariou S. Dissemination and conservation of cadmium and arsenic resistance determinants in Listeria and other Gram-positive bacteria. wiley. 2020;(December 2019):560-9.

68. Guo S, Mahillon J. pGIAK1, a heavy metal resistant plasmid from an obligate alkaliphilic and halotolerant bacterium isolated from the Antarctic Concordia Station confined environment. PLoS One. 2013;8(8):1-8.

69. Pombinho R, Camejo A, Vieira A, Reis O, Carvalho F, et al. Listeria monocytogenes CadC regulates cadmium efflux and fine tunes lipoprotein localization to escape the host immune res- 
ponse and promote infection. J Infect Dis. 2017;215(9):1468-79.

70. Gómez-Sanz E, Kadlec K, Feßler AT, Torres C. Analysis of a novel erm ( T ) - and cadDX - carrying plasmid from methicillin- susceptible Staphylococcus aureus ST398-t571 of human origin. J Antimicrob Chemother [Internet]. 2013;68(October 2012):471-3. Available from: https:// academic.oup.com/jac/article/68/2/471/676191

71. Argudín MA, Hoefer A, Butaye P. Heavy metal resistance in bacteria from animals. Res Vet Sci [Internet]. 2019;122:1-57. Available from: https://doi.org/10.1016/j.rvsc.2018.11.007 72. Chen J, Sun GX, Wang XX, Lorenzo V De, Rosen BP, Zhu YG. Volatilization of arsenic from polluted soil by Pseudomonas putida engineered for expression of the arsM arsenic (III) S-adenosine methyltransferase gene. Environ Sci Technol. 2014;48(17):10337-44.

73. Prabhakaran R, Rajkumar SN, Ramprasath T, Selvam GS. Identification of promoter P $c a d R$, in silico characterization of cadmium resistant gene cadR and molecular cloning of promoter PcadR from Pseudomonas aeruginosa BC15. Toxicol Ind Health. 2018;34(12):819-33.

74. Prabaharan C, Kandavelu P, Packianathan C, Rosen BP, Thiyagarajan S, Biotechnology A. Structures of two ArsR As(III)-responsive transcriptional repressors : implications for the mechanism of derepression. J Struct Biol. 2020;207(2):209-17.

75. Jain S, Bhatt A. Molecular and in situ characterization of cadmium-resistant diversified extremophilic strains of Pseudomonas for their bioremediation potential. 2014;297-304.

76. Stahl A, Pletzer D, Mehmood A, Ullrich MS. Marinobacter adhaerens HP15 harbors two CzcCBA efflux pumps involved in zinc detoxification. Antonie Van Leeuwenhoek. 2015; 8 (6):1-5 77. Mazhar SH, Herzberg M, Fekih I Ben, Zhang C. Comparative insights into the complete genome sequence of highly metal resistant Cupriavidus metallidurans srtrain BS1 isolated from a gold - copper mine. Front Microbiol. 2020; 11(2):1-21

78. Nies DH. The biological chemistry of the transition metal "transportome" of Cupriavidus metallidurans. Cupriavidus. 2016;23-6.

79. Sharma P, Asad S, Ali A. Construction of an Escherichia coli biosensor for $\mathrm{Cd}^{2+}$ and sensitivity analysis by low-temperature induction. J Biosci. 2013;38(2):251-8.

80. Huang CW, Wei CC, Liao VHC. A low cost color-based bacterial biosensor for measuring arsenic in groundwater. Chemosphere [Internet]. 2015;141:44-9. Available from: http://dx.doi. org/10.1016/j.chemosphere.2015.06.011

81. Roointan A, Shabab N, Karimi J, Rahmani A, Alikhani MY, saidijam M. Designing a bacterial biosensor for detection of mercury in water solutions. Turkish J Biol. 2015;39(4):550-5.

82. Tynecka Z, Malm A, Gos Z. $C d^{2+}$ extrusion by P-type $C d^{2+}$-ATPase of Staphylococcus aureus $17810 \mathrm{R}$ via energy-dependent $C d^{2+/ H+}$ exchange mechanism. 2016;651-63.

83. Branco R, Cristóvão A, Morais P V. Highly sensitive, highly specific whole-cell bioreporters for the detection of chromate in environmental samples. PLoS One. 2013;8(1):13-7.

84. Li R, Wu H, Ding J, Li N, Fu W, Gan L, et al. Transgenic merA and merB expression reduces mercury contamination in vegetables and grains grown in mercury contaminated soil. Plant Cell Rep. 2020;39 (10):1369-80.

85. Zhou X, Li J, Wang W, Yang F, Fan B, Zhang C, et al. Removal of chromium (VI) by Escherichia coli cells expressing cytoplasmic or surface-displayed chrB: A comparative study. J Microbiol Biotechnol. 2020;30(7):996-1004.

86. Xu S, Sun B, Wang R, He J, Xia B, Xue Y, et al. Overexpression of a bacterial mercury transporter MerT in Arabidopsis enhances mercury tolerance. Biochem Biophys Res Commun [Internet]. 2017;490(2):528-34. Available from: http://dx.doi.org/10.1016/j.bbrc.2017.06.073 